\title{
Determining the Change in Length of the Anterolateral Ligament During Knee Motion: A Three-Dimensional Optoelectronic Analysis.
}

Thomas Neri, MD, PhD ${ }^{1,2,3 *}$

Rodolphe Testa, $\mathrm{PhD}^{1}$

Loic Laurendon, $\mathrm{MD}^{2}$

Margaux Dehon ${ }^{2}$

Sven Putnis, $\mathrm{MD}^{3}$

Samuel Grasso, $\mathrm{PhD}^{3}$

David A Parker, $\mathrm{MD}^{3}$

Frederic Farizon MD ${ }^{1,2}$

Remi Philippot, MD, PhD ${ }^{1,2}$

${ }^{1}$ Univ Lyon - UJM-Saint-Etienne, Inter-university Laboratory of Human Movement Science, EA 7424, F-42023, Saint-Etienne, France

${ }^{2}$ Department of Orthopedic Surgery, University Hospital of Saint Etienne, France

${ }^{3}$ Sydney Orthopaedic Research Institute, Sydney, Australia

*corresponding author: thomas.neri@chu-st-etienne.fr, Inter-university Laboratory of Human Movement Science, CAMPUS SANTE INNOVATIONS Bâtiment IRMIS, 10 rue de la

Marandière, 42270 Saint Priest en Jarez, +33477421875

\section{COMPLIANCE WITH ETHICAL STANDARDS}

Conflict of Interest: The authors declare that they have no conflict of interest.

Funding: This research did not receive any specific grant from funding agencies in the public, commercial, or not-for-profit sectors.

Ethical approval: This article does not contain any studies with human participants or animals performed by any of the authors.

ABSTRACT: 234 words

MANUSCRIPT: 2596 words 


\section{ABSTRACT}

Background: The variation of the anterolateral ligament (ALL) length during knee motion is still unclear, and the knee position in which a reconstruction graft should be tensioned remains controversial. The objective of this study was to determine the variation of the ALL length during knee motion using a three-dimensional optoelectronic system.

Methods: Kinematic analyses of 20 cadaveric knees were performed using a Motion Analysis ${ }^{\circledR}$ system. The variability of the measurements made during the five acquisition cycles was studied. Reliability was evaluated by two separate measurement sessions, with complete system reinstallation, using different cadavers and a new operator. The ALL length was analysed from extension to full flexion in three rotational conditions.

Findings: When analysing the reliability of the five cycles, $82 \%$ of the measurements we found to have an Intra Class Correlation (ICC) $>0.85$. The reproducibility of inter-sessional measures by different operators and different cadavers was either good (ICC >0.75) or excellent (ICC >0.85). The ALL length was maximum in full internal rotation with the knee at $25^{\circ}$ of flexion.

Interpretation: This three-dimensional optoelectronic protocol allowed us to analyse the variation of the ALL length during intact knee motion with good reliability and the required accuracy to analyse this variable. The maximal length and highest tension of the ALL was reported at $25^{\circ}$ of knee flexion in internal rotation, suggesting this as the optimal position for the knee joint when tensioning an ALL reconstruction.

KEYWORDS: knee, Anterolateral ligament, kinematic, length, optoelectronic system 


\section{INTRODUCTION}

Good control of rotational stability after intra-articular anterior cruciate ligament (ACL) reconstruction is not always achieved and there has been a renewed interest in the role of extra-articular structures, among them the anterolateral ligament (ALL) (Claes et al., 2013; Neri et al., 2018a). Combined ACL and ALL reconstructions in ACL deficient knee have been suggested to offer clinical and biomechanical advantages in controlling anterolateral rotational laxity more than an isolated ACL reconstruction in ACL-deficient knee (Geeslin et al., 2018b; Sonnery-Cottet et al., 2015). During ALL reconstruction it is necessary to fix the graft in a position close to its maximum length, corresponding to its range of action, in order to restore normal biomechanics, and avoid insufficient tension or overconstraint. To date there is no consensus on this point and different surgical techniques have subsequently appeared, fixing the graft either with the knee in full extension (Sonnery-Cottet et al., 2015), at $30^{\circ}$ of flexion (Chahla et al., 2016), or at $90^{\circ}$ of flexion (Helito et al., 2015). A detailed understanding of the biomechanical behaviour of the ALL is therefore required to facilitate an optimal reconstruction technique.

The first studies undertaken to evaluate the variation of the ALL length were static analyses using digital calipers measurements at predetermined flexion and rotational values (Claes et al., 2013; Neri et al., 2017; Runer et al., 2016). Three-dimensional imaging analyses have also been used (Helito et al., 2014; Kernkamp et al., 2016; Van de Velde et al., 2016; Wieser et al., 2017). Although having the advantage to be performed on in vivo subjects, these models were created from theoretical insertions points, which are difficult to identify on MRI and subject to significant inter-individual variability (Daggett et al., 2016; Neri et al., 2018b; Parker and Smith, 2016). Other studies used freedom robotic systems or knee rig systems (Dodds et al., 2014; Drews et al., 2017; Geeslin et al., 2018a; Kittl et al., 2015; Parsons et al., 2015). These systems have excellent reliability and optimal precision by dispensing with manipulation by a human operator. Yet all these studies used isolated knees, with sectioning of the musculotendinous structures around the knee, such as the biceps femoris tendon and Iliotibial Band (ITB) subsequently losing their contribution to the rotational stability of the knee and potentially affecting the ALL function (LaPrade et al., 2005; Rahnemai-Azar et al., 2016). Surgical navigation systems designed for prosthetic knee surgery have also been used with the advantage of conserving the full leg and the ITB (Bonanzinga et al., 2016; Imbert et al., 2016). However, these studies assessed the ALL length without combining both continuous flexion and rotation kinematics. Internal rotation was applied only for some target flexion values ( 20 and $90^{\circ}$ for Imbert, and 30 and $90^{\circ}$ for Bonanzinga). In addition, these systems use few cameras with lower data acquisition frequencies, making it difficult to assess the biomechanical behaviour of the ALL (Güler et al., 2013).

Consequently, there are contradictions in current biomechanical results when describing the variation in ALL length during motion leading to inconstancies about the position of the knee at which the ALL is at maximum length. We hypothesised that the use of another measurement device, a three-dimensional optoelectronic system, such as the Motion

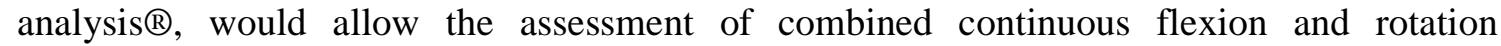
kinematics and lead to an accurate and reliable measurement of the ALL length. The objective of this study was therefore to determine the variation of the ALL length during knee motion. 


\section{METHODS}

\section{Specimen preparation}

Twenty-two intact knees from 11 fresh frozen cadaveric specimens were used. The specimens were thawed out at room temperature for 24 hours and showed no signs of degeneration. Exclusion criteria were examination signs of knee instability (anterior tibial drawer and positive pivot-shift test), evidence of prior knee surgery or ACL reconstruction, severe deformities or severe knee osteoarthritis. An anteromedial knee arthroscopy was performed to confirm the Anterior Cruciate Ligament (ACL) status. A total of two knees were subsequently excluded and 20 intact knees without ACL and ALL injuries were included. There were 5 men and 5 women with a mean age of 68.9 years (range, 57 to 85).

We used the full leg and pelvis in order to preserve the entire length of the ITB and all other synergistic bi-articular structures crossing the hip and/or the knee. The dissection protocol used to define the ALL was described in a previous anatomical study (Neri et al., 2017). The number of incisions was kept to the strict minimum in order to limit their effects, and they were always made in line with the tendinous fibres. No lateral structures were removed. The femoral origin of the ALL was always posterior and proximal to the lateral femoral epicondyle and its tibial insertion was posterior to Gerdy's tubercle, anterior to the fibular head and distal to the articular cartilage of the lateral tibial plateau. At the end of the dissection, once the insertions were recorded and the acquisitions made, the ITB was anatomically closed.

\section{Experimental set-up}

Superior acetabular screws fixed the pelvis to the table and the cadavers were positioned to allow free range of motion of the knee over the edge of the table (Figures 1B and 1C).

Kinematic analysis was performed using a Motion Analysis ${ }^{\circledR}$ (Motion Analysis corp., Santa Rosa, CA, USA) stereophotogrammetry system. The system consisted of 8 high-definition Raptor-E® cameras operating at $100 \mathrm{~Hz}$ (Figure 1A). After installation and calibration around the working area the system followed retro-reflective sensors (Targets). A pelvic marker was defined from 3 targets fixed on the ipsilateral anterior superior iliac spine. The femur and the tibia were equipped with 4 targets each: F1 to F4 and T1 to T4 (Figure 2). These targets were fixed using bi-cortical pins placed in such a way as to leave free the muscles and ligaments. Three points are sufficient to reconstruct the movements of a solid in space, and the use of the fourth provided a backup in the event of disengagement of a target or temporary masking. Using a navigation probe, points of interest were next identified. The epicondyles and malleoli were then calculated (Figure 2, purple stars) from these palpated points (Figure 2, purple circles). The centre of the hip was calculated kinematically via circumduction (Gamage and Lasenby, 2002) to overcome the hip movement during knee motion (Figure 2, purple star). The centre of Inter Condylar Eminences (ICE) was located arthroscopically. The set of points determines the axes and the femoral and tibial references as defined in ISB conventions and the work of Grood and Suntay (Grood and Suntay, 1983; Wu et al., 2002). The centre of the tibial and femoral insertion points of the ALL were identified by internally rotating the tibia and determining the course of the central ligamentous fibres coming under the most tension; the position for the optimal surgical reconstruction.

\section{Determining the Change in Length of the ALL}

The study was divided into two separate sessions of 10 knees each, separated by one month and performed by two different operators in order to appreciate the reliability of the experimental process. The knees were different between the two sessions. 
We studied the knee flexion kinematics in three different test conditions: Forced internal rotation (IR) Forced external rotation (ER) and neutral rotation (NR. For NR, the foot was placed in neutral rotation and the tibia in its reduced position with respect to the femur with unconstrained tibial rotation. A dynamometric torque rig triggering at $5 \mathrm{~nm}$, placed above the ankle at the axis of rotation joint and fixed by 2 pins, provided rotation (Figure 3 ).

The knee was flexed manually by moving the tibia relative to the femur from complete extension to $90^{\circ}$ of flexion while controlling the rotation with the dynamometric torque rig. In every test condition, this movement was repeated five times and performed with a very slow speed of 5 seconds per movement corresponding to an average speed of PI / $10=0.3 \mathrm{Rad} . \mathrm{s}^{-1}$. After processing the kinematics using Cortex ${ }^{\circledR}$ software, the data was filtered (Butterworth filter of order 4 with a cut off frequency of $6 \mathrm{~Hz}$ ) according to Winter and Pezzack (Pezzack et al., 1977; Winter et al., 1974). The recorded data was interpolated to obtain values from full extension to $90^{\circ}$ of flexion at each degree of flexion. Therefore, we could determine the internal-external rotation angle (ROT) and the distance between the femoral and tibial insertions of the ALL (ALL length) during the full range of knee motion (Figure 4).

\section{Statistical analysis}

All statistical analyses were performed using SPSS ${ }^{\circledR}$ software (IBM, Armonk, New York, United States).

Initial statistical analysis was for reliability of the five cycles during the kinematic acquisitions for the three conditions of rotation (IR, ER and NR) and for both variables of interest (ROT, ALLlength). This analysis included all the knees ( $\mathrm{n}=20$ knees). A statistical test of the intraclass correlation coefficient (ICC) was used for each measured variable (ROT, ALLlength). According to Smith-Crowe et al., ICC was considered good if it was $\geq 0.75$ and excellent if it was $\geq 0.85$ (Smith-Crowe et al., 2013). For accepting data without modification, a threshold of 0.85 was required. Two-way mixed ICC calculations with an absolute agreement search were performed. A second statistical analysis was performed to evaluate the reliability of our protocol between the two separate measurement sessions (for each session, $\mathrm{n}=10$ knees). Using the statistical method described above, we calculated the mean curves of the measurements (ROT, ALLlength) during the two sessions for the three test conditions (IR, ER and NR). The ICC of these average curves was then calculated from the data from these two sessions (two-way randomized ICC). In order to compare the lengths of ALL between the IR, NR and ER, a variance analysis study (ANOVA) was performed on repeated measurements. P values less than 0.05 were considered statistically significant. 
RESULTS

\section{Accuracy and Reliability}

The average error of target positioning was consistently less than $0.15 \mathrm{~mm}$ and the measurement error of the angles was less than 0.2 degrees. In the first analysis on the reliability of the five cycles, ICCs were performed for 120 measurements. $82 \%$ of these values had an ICC $>0.85$ and did not require curve suppression. $15 \%$ required the removal of one curve to obtain an ICC $\geq 0.85 .3 \%$ required the removal of 2 curves. In one case, the worst, the ICC after removal of 2 curves was 0.76 . Figure 5 illustrates the variability of the measurements (acquisitions) for a knee.

The second analysis showed either good or excellent reproducibility between the two sessions with different operators and different knees. For the ROT measurement, the ICC was 0.93 in IR and 0.83 in ER. For the ALL length measurement, the ICCs were 0.86 in IR, 0.99 in ER, and 0.98 in NR.

207

Determining the Change in Length of the ALL

After determination of the femoral and tibial insertion points of the ALL, it was then possible to measure the length of the ALL throughout the full range of motion. This length was studied for the 3 rotational conditions in order to determine the flexion and rotation conditions for which the ALL was overtight (maximum length). There was a significant difference for ALL length between the IR and the NR rotation $(p<0.001)$, whatever the knee flexion (Figure 6). In contrast, there was no significant difference between NR and ER ( $p>0.05)$. The ALL length was maximum in IR at $25^{\circ}$ of knee flexion. It should be noted that there is considerable variation in ALL length between individual knee specimens, which is indicated in figure 6 as the extended error bars for the ALL length at different degrees of knee flexion. 


\section{DISCUSSION}

By using an accurate and reliable three-dimensional optoelectronic system, we were able to analyse the variation in length of the ALL during motion in an intact knee. We demonstrated that its maximum length was in IR at $25^{\circ}$ of knee flexion.

Compared to other experimental tools, such as robotic, 3D scan model and traditional navigation systems, our protocol was the first to use a stereophotogrammetry system such as Motion analysis ${ }^{\circledR}$, recognized as the current gold standard for an instrument evaluating kinematics in three-dimensions. This measurement tool allowed us to obtain continuous knee kinematics combining flexion and rotation in a full lower limb with all the bi-articular structures conserved. By determining the change in length of the ALL during knee motion, the aim of this study has been fulfilled with the required accuracy to analyse the small change in this variable and with good reproducibility of the kinematic assessments.

Experimental accuracy depends on the type of sensor fixation used, the number and configuration of the cameras used and the size of the work volume. To reduce the incidence of experimental errors, we used a large number of cameras (8 HD cameras), a volume restricted to the maximum $(0.8 \mathrm{~m} / 1.2 \mathrm{~m} / 1.5 \mathrm{~m})$, stable bone fixation, and a wide inter-distance between two targets. The mean error was always less than $0.15 \mathrm{~mm}$ and the angle measurement error was less than 0.2 degrees. This protocol therefore has optimal precision for acquisition of data and far greater than when determining the centre of the anatomical insertions; the ALL insertion points cover areas over $5 \mathrm{~mm}^{2}$ and it is difficult to precisely evaluate the centre. In order to minimize this bias, all dissections were made by an experienced operator who observed the position of the ALL during internal tibial rotation.

Regarding the reproducibility, this protocol demonstrated a good intra-rater reliability between measurements with $82 \%$ of ICCs superior to 0.85 . In addition, this protocol demonstrated either good or excellent inter-sessional reliability when compared with a second session with new cadaveric set-ups and operator. This makes it possible to validate its use in multisession biomechanical studies.

The protocol described in this study will have many clinical applications. In vitro, it will allow the in-depth study of healthy knee kinematics, or after injury of the ACL and anterolateral complex (ALC). The individual functions of structures composing the ALC (ALL, anterolateral capsule, and iliotibial band Kaplan fibres) are still controversial and unclear. Their individual contribution to the anterolateral rotational laxity require an accurate experimental assessment. It can also be applied to the post-operative knee providing a greater understanding of the role of the ALL reconstruction in providing additional rotational control, and how it may alter knee kinematics.

In vivo, this study provides a useful information to guide ALL reconstruction that may be required in primary surgery for patients with a combined ACL and ALC injury as well as those requiring revision surgery after a first failed ACL reconstruction (Sonnery-Cottet et al., 2017, 2015). Although this additional procedure has shown clinical and biomechanical benefits (Geeslin et al., 2018b; Sonnery-Cottet et al., 2015), there are still inconsistencies in graft fixation. In order to ensure efficient and physiological biomechanical behaviour, the graft has to be fixed close to the range of flexion where the ALL operates, i.e when it is tensioned. To date, there is no clear consensus on this point. Regardless of measurement and instrumentation factors, the literature suggests that the other main factor influencing the length is the location of the ALL femoral origin (Monaco et al., 2017). Helito et al., recommends that the graft is fixed with the knee flexed between 60 and $90^{\circ}$ (Helito et al., 2015, 2013) with the ALL femoral footprint identified either anterior or on the lateral femoral 
epicondyle. Sonnery-Cottet et al., recommend graft fixation close to extension and neutral rotation to avoid overtightening in external rotation (Sonnery-Cottet et al., 2015). They located the ALL femoral origin posterior to the epicondyle. In another study, the same team demonstrated that the ALL is tight when the knee is at $20^{\circ}$ of flexion with internal rotation of the tibia (Imbert et al., 2016). This is similar to Chahla et al. who recommend graft fixation at $30^{\circ}$ of flexion (Chahla et al., 2016). We also found the ALL femoral origin to be in a posterior and proximal position (Neri et al., 2017), and proved it is at its longest with the knee at $25^{\circ}$ of flexion, suggesting that this is the most appropriate position to have the knee in when tensioning a reconstruction.

Several limitations should be noted. Firstly, the small sample size and the variations between the individual knees and specimens has contributed to the large range in ALL length seen. With 20 knees studied, our sample size is however larger than the majority of ALL biomechanical studies with an average of 10 knees. Secondly, we did not use a rig to bend the knee with the knee range of motion performed manually. Nonetheless, the intra-rater reliability was good to excellent and the movement was performed with a low speed. This slow motion allowed to overcome the effects of speed and therefore recalculation of the angles and positions in a static model by interpolating them at each degree of flexion without using angular velocities.

287

288

289

290

291

292

293

294

295

296

297

298

299

300

301

302

303

\section{CONCLUSION}

This three-dimensional optoelectronic protocol allowed us to analyse the variation of the ALL length during knee motion with good reliability and the required accuracy to analyse this variable. This makes it a valuable protocol that can be used when carrying out future biomechanical analyses necessary to optimise ALL reconstruction techniques. The maximal length and highest tension of the ALL was reported at $25^{\circ}$ of knee flexion in internal rotation, suggesting this as the optimal position for the knee joint when tensioning an ALL reconstruction. 


\section{REFERENCES}

Bonanzinga, T., Signorelli, C., Grassi, A., Lopomo, N., Bragonzoni, L., Zaffagnini, S., Marcacci, M., 2016. Kinematics of ACL and anterolateral ligament. Part I: Combined lesion. Knee Surg. Sports Traumatol. Arthrosc. Off. J. ESSKA. https://doi.org/10.1007/s00167-016-4259-y

Chahla, J., Menge, T.J., Mitchell, J.J., Dean, C.S., LaPrade, R.F., 2016. Anterolateral Ligament Reconstruction Technique: An Anatomic-Based Approach. Arthrosc. Tech. 5, e453-457. https://doi.org/10.1016/j.eats.2016.01.032

Claes, S., Vereecke, E., Maes, M., Victor, J., Verdonk, P., Bellemans, J., 2013. Anatomy of the anterolateral ligament of the knee. J. Anat. 223, 321-328. https://doi.org/10.1111/joa.12087

Daggett, M., Ockuly, A.C., Cullen, M., Busch, K., Lutz, C., Imbert, P., Sonnery-Cottet, B., 2016. Femoral Origin of the Anterolateral Ligament: An Anatomic Analysis. Arthrosc. J. Arthrosc. Relat. Surg. Off. Publ. Arthrosc. Assoc. N. Am. Int. Arthrosc. Assoc. 32, 835-841. https://doi.org/10.1016/j.arthro.2015.10.006

Dodds, A.L., Halewood, C., Gupte, C.M., Williams, A., Amis, A.A., 2014. The anterolateral ligament: Anatomy, length changes and association with the Segond fracture. Bone Jt. J. 96-B, 325-331. https://doi.org/10.1302/0301-620X.96B3.33033

Drews, B.H., Kessler, O., Franz, W., Dürselen, L., Freutel, M., 2017. Function and strain of the anterolateral ligament part I: biomechanical analysis. Knee Surg. Sports Traumatol. Arthrosc. Off. J. ESSKA. https://doi.org/10.1007/s00167-017-4472-3

Gamage, S.S.H.U., Lasenby, J., 2002. New least squares solutions for estimating the average centre of rotation and the axis of rotation. J. Biomech. 35, 87-93.

Geeslin, A.G., Chahla, J., Moatshe, G., Muckenhirn, K.J., Kruckeberg, B.M., Brady, A.W., Coggins, A., Dornan, G.J., Getgood, A.M., Godin, J.A., LaPrade, R.F., 2018 . Anterolateral Knee Extra-articular Stabilizers: A Robotic Sectioning Study of the Anterolateral Ligament and Distal Iliotibial Band Kaplan Fibers. Am. J. Sports Med. 46, 1352-1361. https://doi.org/10.1177/0363546518759053

Geeslin, A.G., Moatshe, G., Chahla, J., Kruckeberg, B.M., Muckenhirn, K.J., Dornan, G.J., Coggins, A., Brady, A.W., Getgood, A.M., Godin, J.A., LaPrade, R.F., 2018 b. Anterolateral Knee Extra-articular Stabilizers: A Robotic Study Comparing Anterolateral Ligament Reconstruction and Modified Lemaire Lateral Extraarticular Tenodesis. Am. J. Sports Med. 46, 607-616. https://doi.org/10.1177/0363546517745268

Grood, E.S., Suntay, W.J., 1983. A joint coordinate system for the clinical description of three-dimensional motions: application to the knee. J. Biomech. Eng. 105, 136144.

Güler, Ö., Perwög, M., Kral, F., Schwarm, F., Bárdosi, Z.R., Göbel, G., Freysinger, W., 2013. Quantitative error analysis for computer assisted navigation: a feasibility study. Med. Phys. 40, 021910. https://doi.org/10.1118/1.4773871

Helito, C.P., Bonadio, M.B., Gobbi, R.G., da Mota E Albuquerque, R.F., Pécora, J.R., Camanho, G.L., Demange, M.K., 2015. Combined Intra- and Extra-articular Reconstruction of the Anterior Cruciate Ligament: The Reconstruction of the Knee Anterolateral Ligament. Arthrosc. Tech. 4, e239-244. https://doi.org/10.1016/j.eats.2015.02.006

Helito, C.P., Demange, M.K., Bonadio, M.B., Tírico, L.E.P., Gobbi, R.G., Pécora, J.R., Camanho, G.L., 2013. Anatomy and Histology of the Knee Anterolateral Ligament. 
Orthop. J. Sports Med. 1, 2325967113513546.

https://doi.org/10.1177/2325967113513546

Helito, C.P., Helito, P.V.P., Costa, H.P., Bordalo-Rodrigues, M., Pecora, J.R., Camanho, G.L., Demange, M.K., 2014. MRI evaluation of the anterolateral ligament of the knee: assessment in routine 1.5-T scans. Skeletal Radiol. 43, 1421-1427. https://doi.org/10.1007/s00256-014-1966-7

Imbert, P., Lutz, C., Daggett, M., Niglis, L., Freychet, B., Dalmay, F., Sonnery-Cottet, B., 2016. Isometric Characteristics of the Anterolateral Ligament of the Knee: A Cadaveric Navigation Study. Arthrosc. J. Arthrosc. Relat. Surg. Off. Publ. Arthrosc. Assoc. N. Am. Int. Arthrosc. Assoc. 32, 2017-2024. https://doi.org/10.1016/j.arthro.2016.02.007

Kernkamp, W.A., Van de Velde, S.K., Hosseini, A., Tsai, T.-Y., Li, J.-S., van Arkel, E.R.A., Li, G., 2016. In Vivo Anterolateral Ligament Length Change in the Healthy Knee During Functional Activities-A Combined Magnetic Resonance and Dual Fluoroscopic Imaging Analysis. Arthrosc. J. Arthrosc. Relat. Surg. Off. Publ. Arthrosc. Assoc. N. Am. Int. Arthrosc. Assoc. https://doi.org/10.1016/j.arthro.2016.07.008

Kittl, C., Halewood, C., Stephen, J.M., Gupte, C.M., Weiler, A., Williams, A., Amis, A.A., 2015. Length change patterns in the lateral extra-articular structures of the knee and related reconstructions. Am. J. Sports Med. 43, 354-362. https://doi.org/10.1177/0363546514560993

LaPrade, R.F., Bollom, T.S., Wentorf, F.A., Wills, N.J., Meister, K., 2005. Mechanical properties of the posterolateral structures of the knee. Am. J. Sports Med. 33, 1386-1391. https://doi.org/10.1177/0363546504274143

Monaco, E., Lanzetti, R.M., Fabbri, M., Redler, A., De Carli, A., Ferretti, A., 2017. Anterolateral ligament reconstruction with autologous grafting: A biomechanical study. Clin. Biomech. Bristol Avon 44, 99-103. https://doi.org/10.1016/j.clinbiomech.2017.03.013

Neri, T., Dalcol, P., Palpacuer, F., Bergandi, F., Prades, J.M., Farizon, F., Philippot, R., Peoc'h, M., 2018a. The anterolateral ligament is a distinct ligamentous structure: A histological explanation. The Knee. https://doi.org/10.1016/j.knee.2018.03.012

Neri, T., Palpacuer, F., Testa, R., Bergandi, F., Boyer, B., Farizon, F., Philippot, R., 2017. The anterolateral ligament: Anatomic implications for its reconstruction. The Knee. https://doi.org/10.1016/j.knee.2017.07.001

Neri, T., Parker, D.A., Beach, A., Boyer, B., Farizon, F., Philippot, R., 2018b. Anterolateral Ligament of the Knee: What we Know About its Anatomy, Histology, Biomechanical Properties and Function. Surg. Technol. Int. 33.

Parker, M., Smith, H.F., 2016. Anatomical variation in the anterolateral ligament of the knee and a new dissection technique for embalmed cadaveric specimens. Anat. Sci. Int. https://doi.org/10.1007/s12565-016-0386-2

Parsons, E.M., Gee, A.O., Spiekerman, C., Cavanagh, P.R., 2015. The biomechanical function of the anterolateral ligament of the knee. Am. J. Sports Med. 43, 669-674. https://doi.org/10.1177/0363546514562751

Pezzack, J.C., Norman, R.W., Winter, D.A., 1977. An assessment of derivative determining techniques used for motion analysis. J. Biomech. 10, 377-382.

Rahnemai-Azar, A.A., Miller, R.M., Guenther, D., Fu, F.H., Lesniak, B.P., Musahl, V., Debski, R.E., 2016. Structural Properties of the Anterolateral Capsule and Iliotibial Band 
of the Knee. Am. J. Sports Med. 44, 892-897.

https://doi.org/10.1177/0363546515623500

Runer, A., Birkmaier, S., Pamminger, M., Reider, S., Herbst, E., Künzel, K.-H., Brenner, E., Fink, C., 2016. The anterolateral ligament of the knee: A dissection study. The Knee 23, 8-12. https://doi.org/10.1016/j.knee.2015.09.014

Smith-Crowe, K., Burke, M.J., Kouchaki, M., Signal, S.M., 2013. Assessing Interrater Agreement via the Average Deviation Index Given a Variety of Theoretical and Methodological Problems. Organ. Res. Methods 16, 127-151. https://doi.org/10.1177/1094428112465898

Sonnery-Cottet, B., Daggett, M., Fayard, J.-M., Ferretti, A., Helito, C.P., Lind, M., Monaco, E., de Pádua, V.B.C., Thaunat, M., Wilson, A., Zaffagnini, S., Zijl, J., Claes, S., 2017. Anterolateral Ligament Expert Group consensus paper on the management of internal rotation and instability of the anterior cruciate ligament - deficient knee. J. Orthop. Traumatol. Off. J. Ital. Soc. Orthop. Traumatol. https://doi.org/10.1007/s10195-017-0449-8

Sonnery-Cottet, B., Thaunat, M., Freychet, B., Pupim, B.H.B., Murphy, C.G., Claes, S., 2015. Outcome of a Combined Anterior Cruciate Ligament and Anterolateral Ligament Reconstruction Technique With a Minimum 2-Year Follow-up. Am. J. Sports Med. 43, 1598-1605. https://doi.org/10.1177/0363546515571571

Van de Velde, S.K., Kernkamp, W.A., Hosseini, A., LaPrade, R.F., van Arkel, E.R., Li, G., 2016. In Vivo Length Changes of the Anterolateral Ligament and Related Extraarticular Reconstructions. Am. J. Sports Med. 44, 2557-2562. https://doi.org/10.1177/0363546516651431

Wieser, K., Fürnstahl, P., Carrillo, F., Fucentese, S.F., Vlachopoulos, L., 2017. Assessment of the Isometry of the Anterolateral Ligament in a 3-Dimensional Weight-Bearing Computed Tomography Simulation. Arthrosc. J. Arthrosc. Relat. Surg. Off. Publ. Arthrosc. Assoc. N. Am. Int. Arthrosc. Assoc. https://doi.org/10.1016/j.arthro.2016.11.005

Winter, D.A., Sidwall, H.G., Hobson, D.A., 1974. Measurement and reduction of noise in kinematics of locomotion. J. Biomech. 7, 157-159.

Wu, G., Siegler, S., Allard, P., Kirtley, C., Leardini, A., Rosenbaum, D., Whittle, M., D'Lima, D.D., Cristofolini, L., Witte, H., Schmid, O., Stokes, I., Standardization and Terminology Committee of the International Society of Biomechanics, 2002. ISB recommendation on definitions of joint coordinate system of various joints for the reporting of human joint motion--part I: ankle, hip, and spine. International Society of Biomechanics. J. Biomech. 35, 543-548. 
Figure 1. Installation of Motion analysis ${ }^{\circledR}$ system with 8 High definition cameras (1a), around the specimen. Front (1b) and lateral (1c) pictures of the set-up showing pelvis, femoral and tibia reflective sensors.

444

445

446

447

448

449

450

451

452

453

454

455

456

457

458

459

460

461

462

463

464

465

466

467

468

469

470

471

472

473

474

475

476

477

Figure 2. Definition of bone landmarks and bone axis

- Femur: F1, F2, F3, F4, FHC* (Femoral Head Center), Lateral Epicondyle (LE), Medial Epicondyle (ME), KC* (Knee center)

- Tibia: T1, T2, T3, T4, center of Inter Condylar Eminences (ICE)

- Ankle: Medial malleolar (MM), Lateral malleolar (LM), Ankle Center* (AC)

- Purple circle= palpated landmarks

- Purple star $=$ calculated landmarks

- Femoral axis: XF (in red), YF (in green), ZF (in blue)

- Tibial axis: XT (in red), YT (in green), ZT (in blue)

Figure 3. Dynamometric torque rig used to control the tibial rotation applied. The rig fixation was ensured by 2 extra-articular bi malleolar (distal tibia and fibula) pins. (3A: draw explaining the pins positioning above the joint line of the ankle, 3B: photograph of the rig used)

Figure 4. Example illustrating ALL length analysis during knee motion regarding rotation and flexion of the knee (ALL: anterolateral ligament)

Figure 5. Example illustrating the reproducibility of measurements over five acquisitions from one knee in three conditions (IR, NR, ER).

(ALL: anterolateral ligament, IR: Internal rotation, ER: External rotation, NR: Neutral rotation)

Figure 6. ALL length variation during knee flexion regarding three conditions of rotation (ALL: anterolateral ligament, IR: Internal rotation, ER: External rotation, NR: Neutral rotation) 


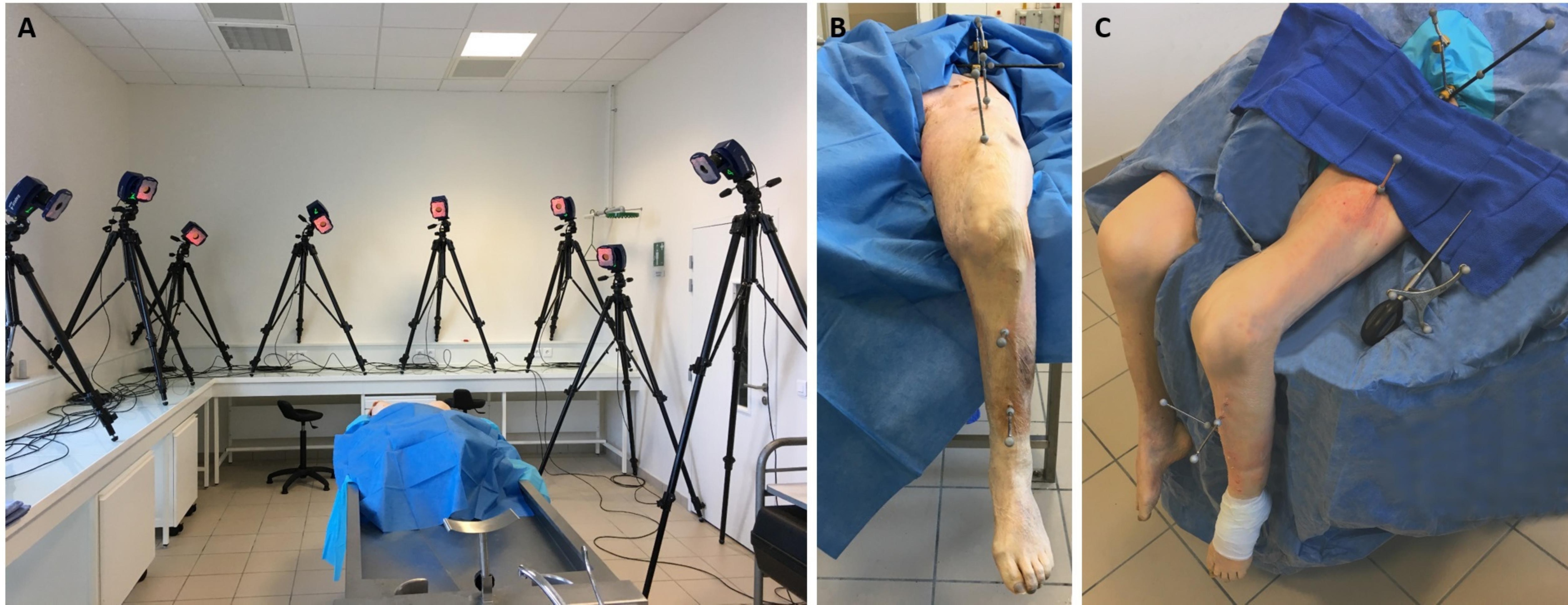




\section{A}

(1) 1

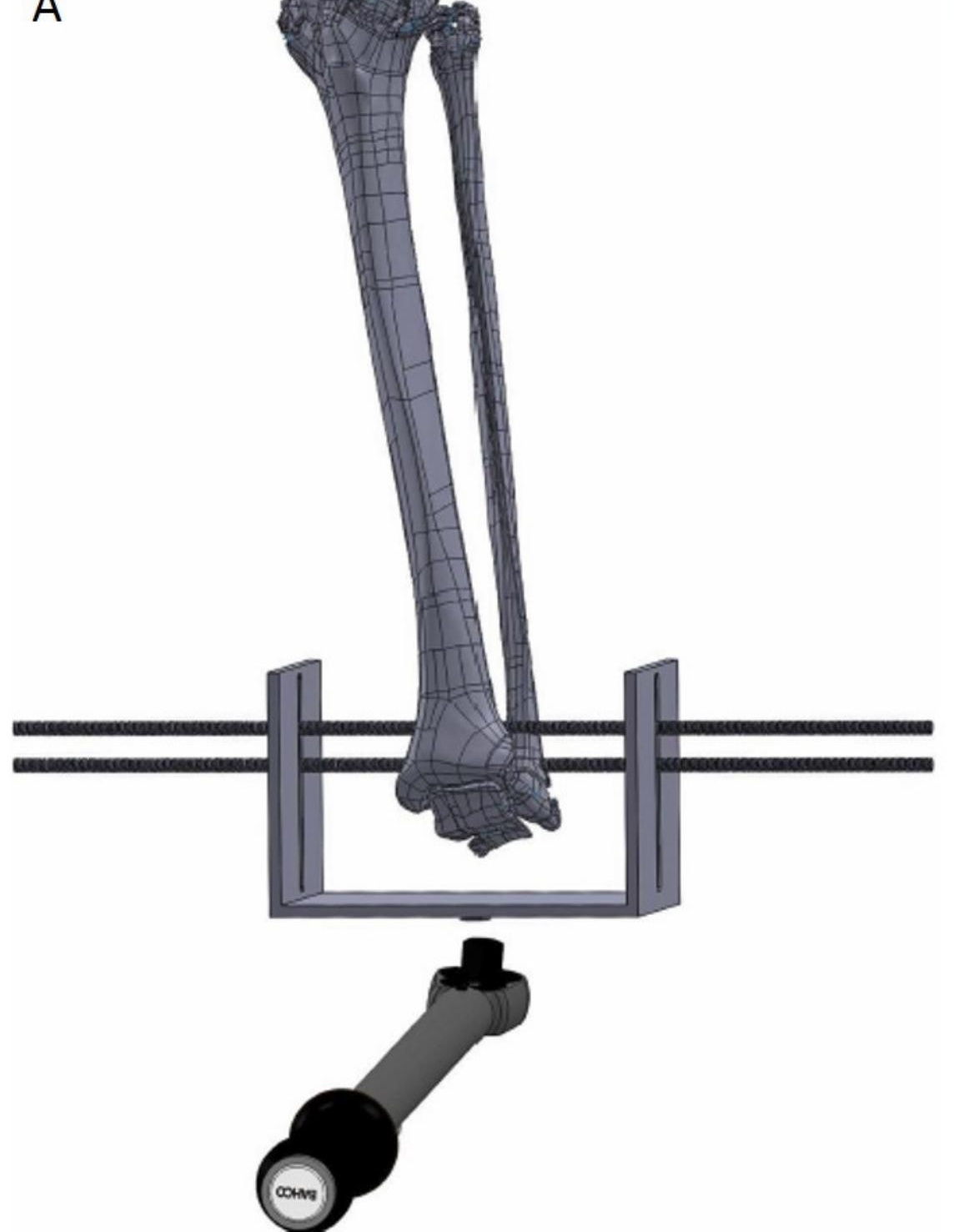

B

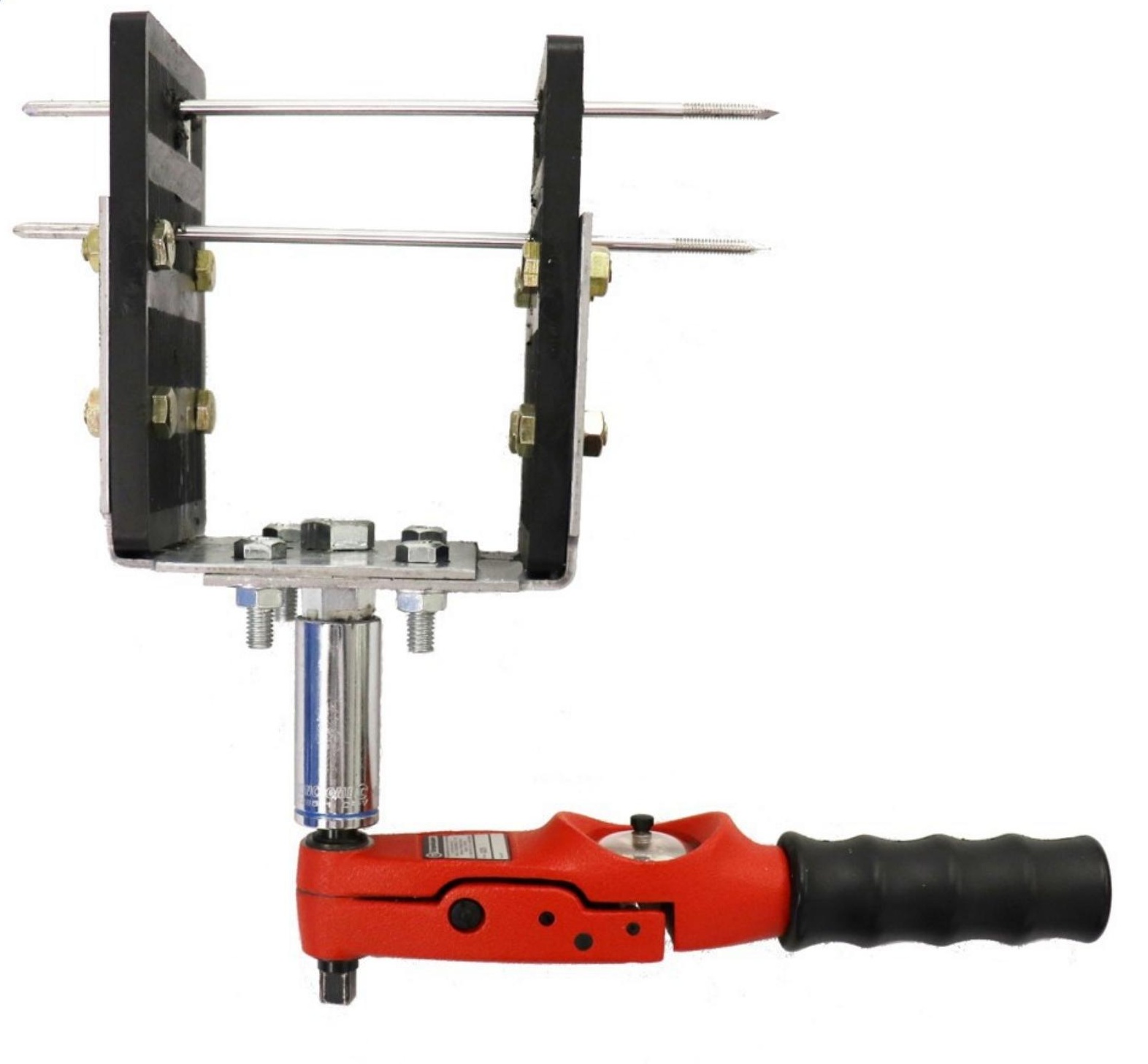


ALL LENGTH (in mm)
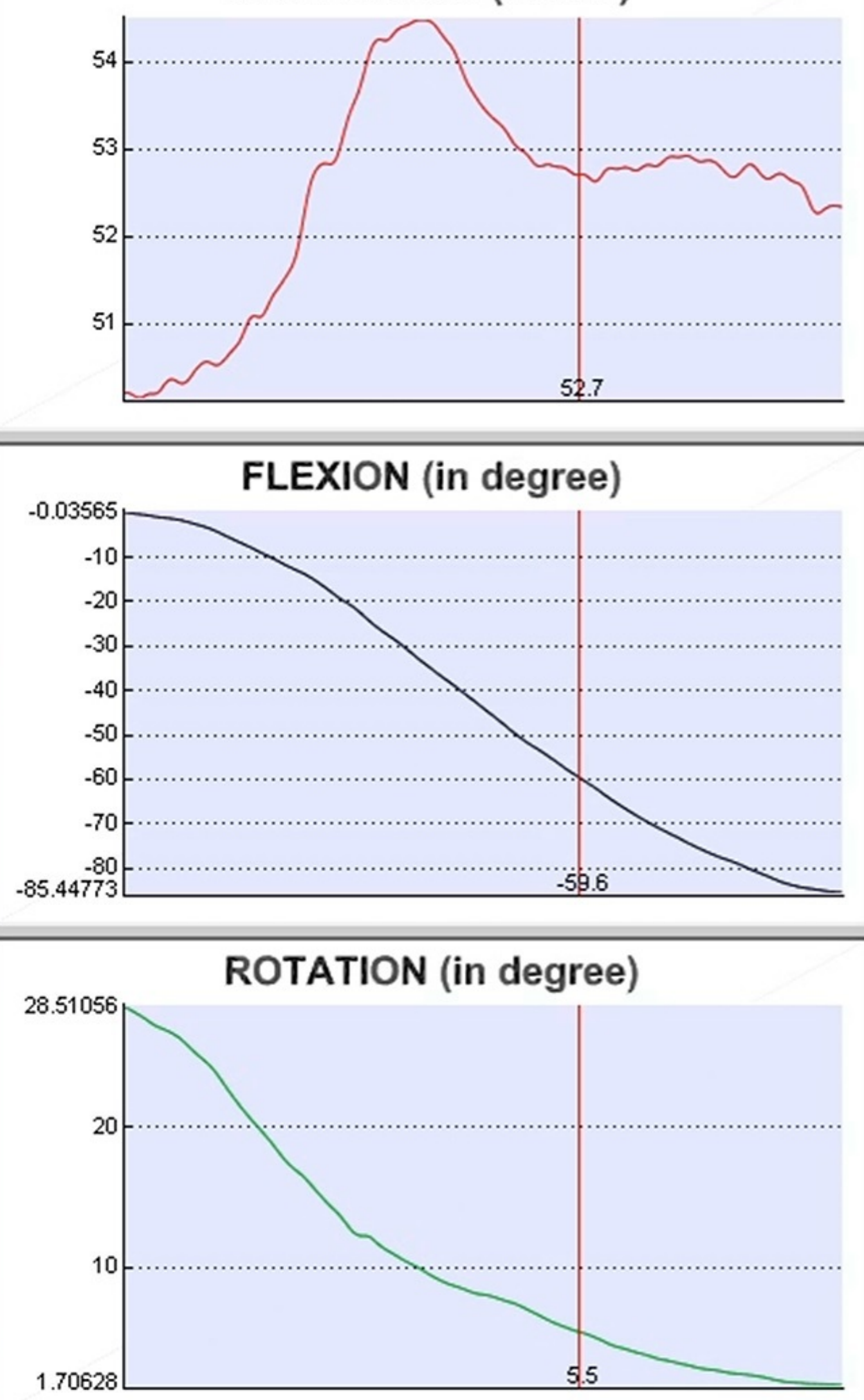
EXTERNAL ROTATION

25

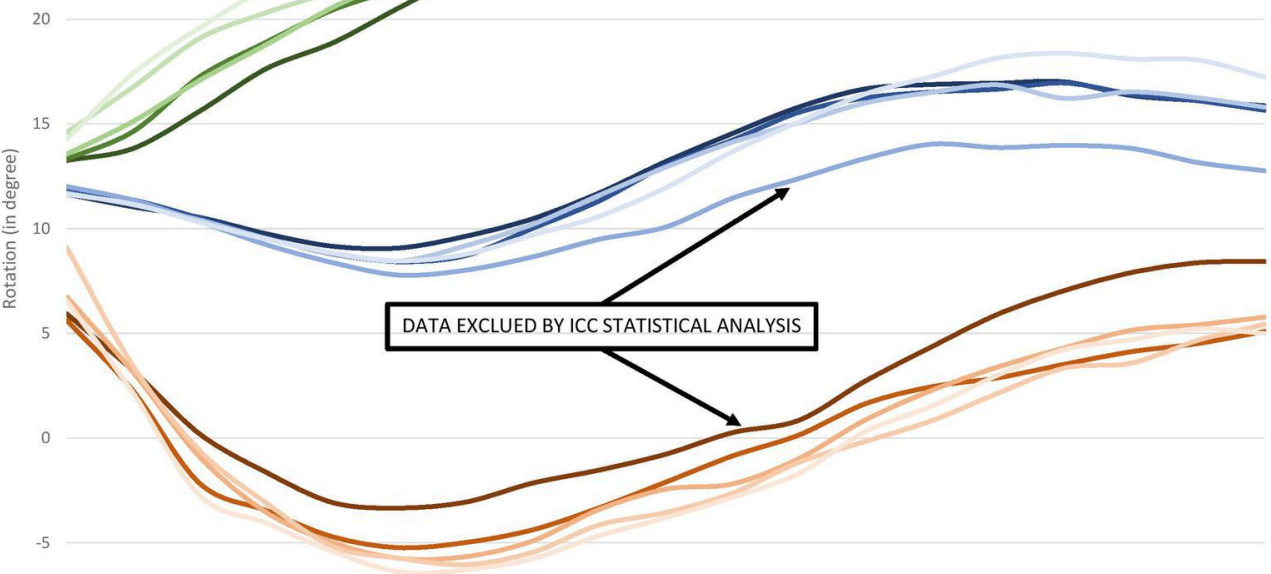

-10 INTERNAL ROTATION

\begin{tabular}{lcccccc}
\hline 0 & 20 & 30 & 40 & 50 & 90 & $70 \quad 90$
\end{tabular}




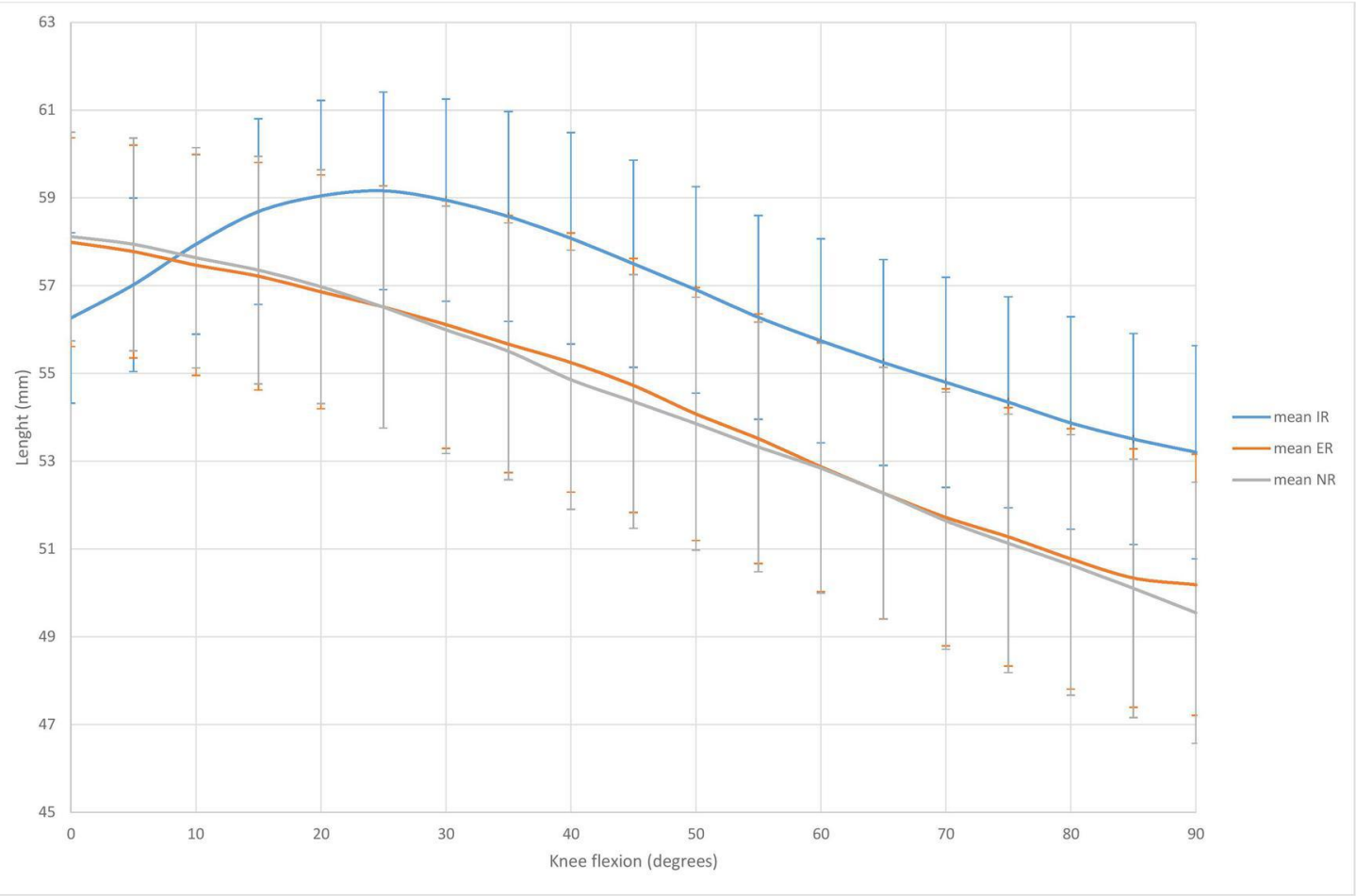

